\title{
Creation and Analysis of Earth's Surface Roughness Maps from Airborne LiDAR Measurements in Downtown Urban Landscape
}

\author{
Fahmy F. F. Asal \\ Civil Engineering Department, Faculty of Engineering, Menoufia University, Shebin El-Kom, Egypt \\ Email: address fahmy_asal@hotmail.com
}

How to cite this paper: Asal, F.F.F. (2019) Creation and Analysis of Earth's Surface Roughness Maps from Airborne LiDAR Measurements in Downtown Urban Landscape. Journal of Geographic Information System, 11, 212-238.

https://doi.org/10.4236/jgis.2019.112015

Received: April 8, 2019

Accepted: April 25, 2019

Published: April 28, 2019

Copyright () 2019 by author(s) and Scientific Research Publishing Inc. This work is licensed under the Creative Commons Attribution International License (CC BY 4.0).

http://creativecommons.org/licenses/by/4.0/

\begin{abstract}
The Earth's surface roughness constitutes an important parameter in terrain analysis for studying different environmental and engineering problems. Authors gave different definitions and measures for the earth's surface roughness that usually depend on exploitation of digital elevation data for its reliable determination. This research aimed at exploring the different approaches for defining and extraction of the Earth's surface roughness from Airborne LiDAR Measurements. It also aimed at evaluating the effects of the window size of the standard deviation filter on the created roughness maps in downtown landscapes using three known approaches namely; standard deviation filtering of the Digital Elevation Model (DEM), standard deviation filtering of the slope gradient model and standard deviation filtering of the profile curvature model. In this context, different roughness maps have been created from Airborne LiDAR measurements of the City of Toronto, Canada using the three filtering approaches with varying window sizes. Visual analysis has shown color tones of small roughness values with smooth textures dominate the roughness maps from small window sizes of the standard deviation filter, however, increasing the window sizes has produced wider variations of the color tones and rougher texture roughness maps. The standard deviations and ranges of the roughness maps from LiDAR DEM have increased due to increasing the filter window size while the skewness and kurtosis have decreased due to increasing the window size, indicating that the roughness maps from larger window sizes are statistically more symmetrical and more consistent. Thus, kurtosis has decreased by $53 \%$ and $82 \%$ due to increasing the window size to $7 \times 7$ and $15 \times 15$ respectively. The standard deviations of the roughness maps from the slope gradient model have increased due to increasing the window size till $15 \times 15$ while they have decreased with more increases. However, skewness has decreased due to increasing the window size
\end{abstract}


till $15 \times 15$ and the kurtosis has decreased with higher rate till window size of $11 \times 11$. In the roughness maps from the profile curvature model, the ranges and skewness have decreased by $93.6 \%$ and $82.6 \%$ respectively due to increasing the window size to $15 \times 15$ while, kurtosis has decreased by $58.6 \%$, $76.3 \%$ and $93.76 \%$ due to increases in the filter window size to $5 \times 5,7 \times 7$ and $15 \times 15$ respectively.

\section{Keywords}

Surface Roughness, Urban Landscape, Airborne LiDAR, DSM/DEM/DTM, Slope Gradient, Profile Curvature, Standard Deviation Filtering, Spatial Analysis

\section{Introduction}

The Earth's surface roughness is an important parameter for terrain analysis as it reflects numerous geophysical parameters such as landform characteristics in addition to playing an important role in analyzing different natural phenomena [1] [2] [3]. Grohmann et al. 2011 \& 2009 [4] [5] defined surface roughness as an expression of the variability of elevations of a topographic surface at a given scale, where the scale of the analysis is determined by the size of the landforms or geomorphic features of interest. Digital Elevation Model (DEM) constitutes an important and objective measure of surface elevation which makes it suitable for representation of the surface features [6] that mainly determine the amount of surface roughness to a specific Engineering or Environmental phenomena [7] [8]. Hodge et al. 2009 [9] carried out investigations of the methods of analyzing digital terrain models through calculating the distribution of surface elevations, semi variograms, surface inclinations, surface slopes and aspects and grain orientation. They recommended that the distribution of surface elevations, surface slope and aspect analysis were found to be most informative. Taud, and Parrot, 2005 [10] studied the relationships between geological features and DEM surface roughness using fractal geometry analysis and recommended that measuring DEM surface roughness represents a helpful tool for morphometric features recognition. Also, Hani et al., 2011 [2] presented an algorithm for computation of the DEM surface roughness that computes the average area of curvature regions in the terrain, and the average roughness of the terrain due to the distribution of curvature regions. They suggested that their algorithm provided realistic surface roughness parameter with respect to amplitudes and frequencies of the terrain that allows good quantification of a region's convexity/concavity over varying scales, however, they did not mention how the type of the landscape could affect their results. Tian et al., 2011 [1] applied quantification of surface-roughness variability using a DEM at Beichuan County before and after an earthquake through exploiting three algorithms namely; root mean square of elevation, standard deviation of elevation, standard deviation of profile curva- 
ture. They recommend that the method of root mean square of elevation showed the most obvious results in depicting changes in the surface roughness before and after the earthquake. Zheng and Zhao 2010 [11] presented a method for estimating surface roughness from radiometer sampling data at different frequencies where their simulated numerical results showed that radiometer with a sensitivity of $0.1 \mathrm{~K}$ can distinguish different surface roughness and changes of roughness.

Grohmann et al. 2011 \& 2009 [4] [5] reviewed the different approaches developed in the study of surface roughness in the earth and planetary sciences followed by assessment of selected methods in rural landscapes in the Midland Valley, Scotland at different scales and spatial resolutions. Their methods include the area ratio method which computes the ratio of actual surface area to the plan area of square cells defined by input points where at flat surfaces this ratio would be close to one, however, with irregular surfaces, the ratio would be bigger than one. Also, they examined the vector dispersion that is an array of regularly spaced elevation values that can be divided into planar triangular surfaces and can be defined by three adjacent non-collinear points and normal to these planes represented by unit vectors where at flat areas with similar elevations the vector strength is expected to be high and the vector dispersion is expected to be small, however, in irregular areas the vector strength will be low and the vector dispersion will be high. Moreover, Grohmann et al. 2011 \& 2009 [4] [5] stated that several authors define surface roughness as the variability of the elevations of earth's surface to be expressed as the absolute standard deviation of all values within user defined window size. In addition to what stated before, the standard deviation of the residual digital elevation model can be exploited as a measure of the surface roughness where the residual DEM is computed through algebraic subtraction of extracted Digital Terrain Model (DTM) from the original DEM representing the actual surface and can referred as the Digital Surface Model (DSM). Furthermore, the standard deviation of slope gradient constitutes an important measure of roughness as it expresses the variability of topography. Additionally, the standard deviation of profile curvature was also used as a measure of surface roughness since curvature constitute the second derivative of the surface elevation and is comprised of three components, profile, planform and tangential curvatures, however, profile curvature, the rate of change of slopes which is the of measure downslope curvature that assists identifying of breaks-of-slope referring to surface roughness [4] [5]. Hebeler and Purves, 2007 [12] exploited high resolution data from the Shuttle Radar Topography Mission in comparison with the GLOBE $1.0 \mathrm{~km}$ data set for calculating error surfaces for three mountainous regions. They recommend that error surfaces are related to a range of topographic variables; roughness, minimum and mean extremity and aspect. They suggested that global statistics for a range of topographic indices are robust to the introduction of uncertainty and topographic indices such as elevation roughness defined as the standard deviation of elevations in a $3 \times 3$ 
neighborhood in addition to the slope roughness expressed as the standard deviation of slopes in a $3 \times 3$ neighborhood are sufficient to study the influence of elevation uncertainty on derivation of such topographic indices [12].

Big advances in computing technology and increasing of storage capacity motivated digital elevation models to be widely used in different applications supported by the availability of high-resolution digital elevation data at horizontal and vertical domains that allows accurate calculations of the parameters extracted from a DEM [8] [9]. In this context, digital terrain analysis can allow extraction of primary attributes such as slope gradient, slope aspect, plan curvature and profile curvature that can be calculated directly from a DEM [7] [13]. This is reflected on the capability of extracting geomorphometric features such as the surface roughness that provide information about regional geomorphology and many roughness parameters since some authors exploited curvature parameters in studying the surface since curvature attributes are helpful in measuring convergence and divergence of the earth's surface [12]. Tay and Teng 2008 [14] stated that image surface roughness is an important characteristic for analyses of InSAR images. They proposed a surface roughness index for estimation of image surface roughness for the applications of InSAR images of different landuse classes and DEMs of different altitudes based on the differences of heights between neighboring pixels within a user defined window. Dinesh 2008 [15] presented a procedure for computing the surface roughness of individual mountainous objects in which mathematical morphology is employed to extract the mountains of the DEM through computing a normalized probability functions for each mountainous object as the ratio of the area of pixels modified in the mountainous object at each scale to the area of the mountainous object and used the computed normalized probability functions to compute the scale-independent average roughness of the mountainous objects due to the distribution of convex and concave regions averaged over the mountainous objects. Korup and Korzeniowska, 2016 [16] estimated surface roughness from LiDAR DEMs as the standard deviation of the total curvature in a fixed search window of size $5 \times 5$ pixels and interpreted this as a measure of terrain roughness.

Digital elevation data derived from new technologies employing active remote sensing methods such as airborne laser scanning and radar ranging are becoming more widespread where past research need to be re-evaluated in the near future to accommodate such new elevation data products and its applications in extraction of different surface parameters such as surface roughness [17] [18] [19]. Airborne Laser Scanning basing on the Light Detection And Ranging (LiDAR) is a very well-established technology that continues developments and providing high spatial resolution terrain coverage with improved accuracy to be utilized in creation of high quality and high accuracy digital elevation models [20] [21] [22]. Frankel and Dolan 2007 [23] state that high-resolution Airborne Laser Swath Mapping (ALSM) which is another acronym for Airborne LiDAR digital topographic data enabled researchers to study topography in unprece- 
dented details allowing them to quantify the earth's surface roughness that was difficult to do in the past. They used ALSM data to calculate surface roughness on two alluvial fans in northern Death Valley, California using their definition of surface roughness as the standard deviation of slopes of a moving window of $5 \times$ 5 size on a DEM. Dorn et al., 2014 [24] carried out a study that focuses on studying surface roughness with exploration of the effects of the roughness layers on the flood simulation results using LiDAR data, orthophotos, official land use data, OpenStreetMap data and CORINE Land Cover data. They applied object-based image analysis to orthophotos and LiDAR DEM in order to generate land cover maps for the purpose of roughness parameterization. Additionally, they exploited vertical vegetation structure within the LiDAR point cloud for extraction of roughness maps in the floodplains with creation of roughness maps from official land use data, OpenStreetMap and CORINE Land Cover datasets where, they applied six different flood simulations basing on one elevation data but within the different roughness maps. They acknowledged that roughness maps derived from LiDAR data and OpenStreetMap data are comparable whereas the results of the other datasets differ significantly. Lia et al., 2016 [25] proposed a non-rasterization method for road lines' surface roughness extraction from LiDAR data basing on the characteristics of airborne LiDAR technology that can give accurate and georeferenced sets of dense point clouds accompanied with the intensity of the returned signals. Airborne LiDAR enjoys several advantages with respect to automatic object extraction compared to aerial imagery that can be as follows [20] [25] [26]:

1) It is much easier to separate tall objects from roads with the use of LiDAR.

2) Surface roughness can be easily obtained from LiDAR data.

3) Airborne LiDAR data from narrow scanning angle and active sensing technology suffers less occlusions and smaller shadows allowing features such as roads to be more complete compared to that from the imageries.

4) The intensity of the reflectance of LiDAR measurements can provide useful means for road extraction since road surfaces should have similar reflectance.

5) Rivers are easy to be detected from LiDAR measurements since water steams absorb laser light to be represented as no-data.

Fan and Atkinson 2018 [20] noticed that the rate of change in the DEM errors is statistically greater in local areas where the surface is rougher when different source data densities are used in creating the DEM with the same degree of data reduction is applied to a whole area. They based on this notice to characterize surface roughness from the differences between two digital elevation models generated using point clouds of different spatial resolutions for the same terrain surface and introduced an approach for estimating surface roughness where they used numerical experiments to test their approach on datasets of four elevation point clouds obtained from terrestrial laser scanning (TLS) and airborne LiDAR. They recommended that their approach is effective in quantifying local terrain surface roughness, however they did not state which type of terrain the approach could work better. 


\section{Research Aims and Objectives}

This research aimed at exploring the different approaches for defining and extraction of the Earth's surface roughness from digital elevation data in general. However, great emphasis has been given to extraction and analysis of surface roughness maps from Airborne LiDAR Measurements in urban landscapes characterized by intensive varieties of different geometry, size, shape and elevation features expected to provide great roughness to resist water movements during flooding. This is added to the wide involvements of the Earth's surface roughness in many other applications. The study also, aimed at evaluating the effects of the size of the user defined window of the standard deviation filtering process on the created roughness maps from airborne LiDAR data in urban downtown landscape using three known approaches for defining and measuring the surface roughness namely; the standard deviation of the surface elevations, the standard deviation of the surface slope gradients and the standard deviation of the surface profile curvatures.

\section{Test Site, Test Data and Methodology}

A sample of LiDAR data of the ISPRS WG III/4 Test Project on Urban Classification and 3D Building Reconstruction has been provided by ISPRS WG III/4. The Optech airborne laser-scanner ALTM-ORION M captured the airborne laser scanning data for a limited area at the city center of the City of Toronto in Canada [26]. The sample data constitutes downtown data of a mixture of lowand high-story buildings with a wide variety of rooftop structures in addition to street and road features see Figure 1. Optech corporation flew over the "Downtown Toronto" area and acquired ALS data using Optech's ALTM-ORION M in February 2009 where the ALTM ORION M operates at a wavelength of 1064 $\mathrm{nm}$ (Near Infrared) and scans the underlying topography with a scan width of 20 degrees and the scan frequency of $50 \mathrm{~Hz}$. The whole dataset consists of 6 strips with point density of approximately 6.0 points $/ \mathrm{m}^{2}$. The data is formatted in ASPRS (American Society of Photogrammetry and Remote Sensing)'s LAS 1.3 format [26]. In this study, the file Toronto_Strip_03.las has been used for creation and analysis of Earth's surface roughness maps from Airborne LiDAR Measurements in Urban Landscape. Toronto_Strip_03.las test data file represents a complete Airborne laser scanning (ALS) strip and covers an area of about $717,296 \mathrm{~m}^{2}$ of approximate dimensions of about 508 meters in swath width by about 1412 meters as the swath length. The sample data consists of $2747785 \mathrm{Li}$ DAR data measurements giving LiDAR point cloud density of 3.83074 points per one meter squared $\left(\mathrm{pts} / \mathrm{m}^{2}\right)$. This means that one LiDAR measurement has been recorded for every 0.261045 squared meters in average. The statistical analysis of the data set showed a minimum elevation of -9.69 meters and a maximum elevation of 165.02 meters giving a range of elevations of 174.71 meters as shown by the legend in Figure 1. Additionally, the mean elevation of the dataset file has recorded of 74.02371 meters. Moreover, the standard deviation of 
the elevations across the downtown test area is 45.49852 meters which refers to considerable changes in the elevations of the buildings and the urban landscape features.

A digital elevation model has been created from the Toronto_Strip_03.las LiDAR data file using SAGA 6.4 open source GIS software where the Inverse Distance Weighting (IDW) interpolation technique with the power of four and grid resolution of half a meter have been used. In addition, a slope gradient model and a profile curvature model have been extracted from the generated DEM of the downtown of the City of Toronto in Canada. Also, each of the generated DEM, slope gradient model and the profile curvature model have been subject to standard deviation filtering of varied window sizes for the production of different surface roughness maps from some of the selected popular surface roughness measures. In this study, standard deviation filters of windows of sizes of $3 \times 3,5 \times 5,7 \times 7,9 \times 9,11 \times 11,15 \times 15,21 \times 21,25 \times 25,31 \times 31,35 \times 35$ and $41 \times 41$ have been used under the surfer 15 commercial software package. The generated surface roughness maps extracted from the standard deviation filtering of elevations, the standard deviation filtering of slope gradient and the standard deviation filtering of profile curvature at varying filter window sizes have been subjected to visual and statistical analysis for the purpose of studying of the effects of the standard deviation filter window size on the extracted roughness maps from Airborne LiDAR measurements in downtown urban landscape.

\section{Creation and Analysis of Surface Roughness Maps from Standard Deviation Filtering of LiDAR DEM}

Figures 2-9 depict surface roughness maps created as a result of standard deviation filtering of Airborne LiDAR DEM using filters of varied window sizes of $3 \times$ $3,5 \times 5,7 \times 7,11 \times 11,15 \times 5,21 \times 21,31 \times 31$ and $41 \times 41$ respectively. In Figure 2 the dark blue color tones dominate the roughness map from standard deviation filtering using window size of $3 \times 3$ referring to small roughness at a downtown area with high intensity of varied height features. This refers to neglection of roughness due to important features in the area. Only the edges of the building are represented by very thin lines of bright color tones of higher roughness values. The texture of the roughness map is smooth at the most parts of the roughness map expect at the outer edges of buildings which tend to be rougher, however, smoothing of roughness due to features at big parts of the roughness map is very clear. Figure 3 created using a filter of window size of $5 \times$ 5 is of brighter color tones compared to Figure 2 but still high degree of feature smoothing with smooth tones and smooth textures dominate the roughness map. With increasing the size of the standard deviation filter more structured roughness maps have been obtained using filter sizes of $7 \times 7$ and $11 \times 11$ as depicted in Figure 4 and Figure 5 respectively. In Figure 4 and Figure 5 the amounts of bright blue tones have increased with thicker edges of the buildings and less smoothing of structures. With increasing the sizes of the standard devi- 
ation filters to $15 \times 15$ and $21 \times 21$ more structured roughness maps have been obtained as depicted in Figure 6 and Figure 7 respectively where, the bright blue color tones representing higher values of roughness have increased with rougher textures and clear representation of the roughness due to different height features in the maps. Figure 8 and Figure 9 depict the most structured roughness maps with limited smoothing of features in addition to structured tones and textures reflecting clear varied elevation features dominating the downtown landscape. Also, the edges of the different height features and buildings have been thick which reflects their high contribution to the surface roughness in the intensive landscape area.

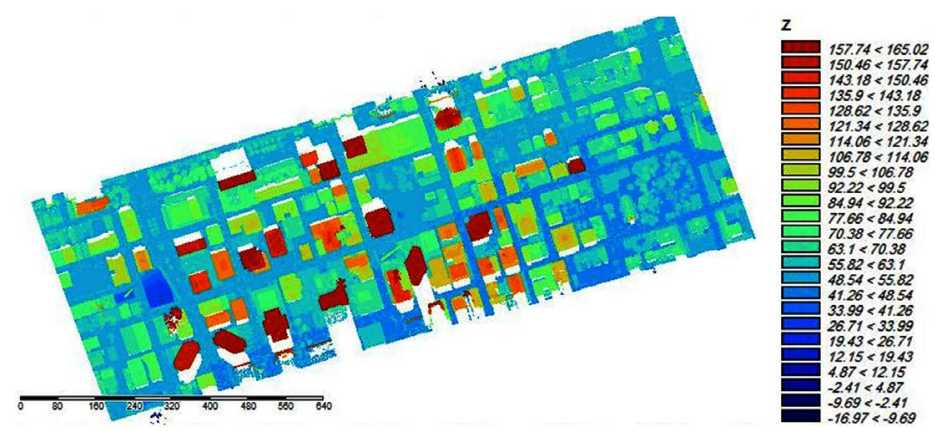

Figure 1. Point cloud Airborne LiDAR dataset for the downtown of the City of Toronto in Canada (elevation $\mathrm{z}$ is in meters).

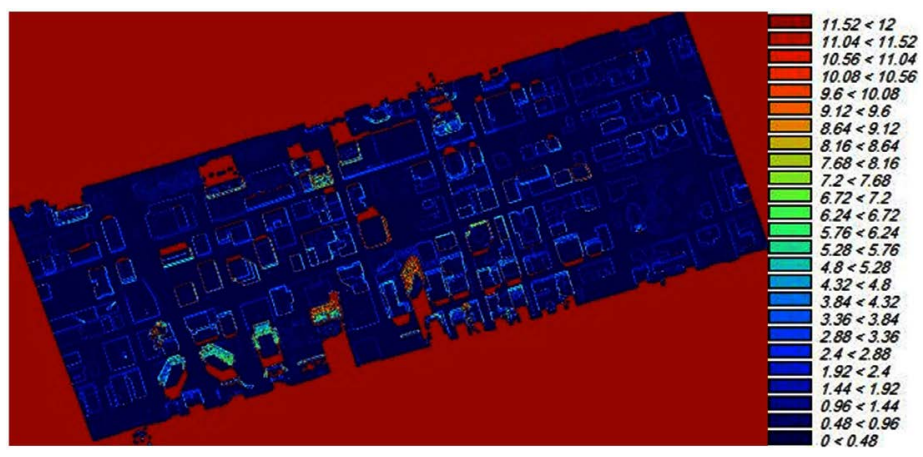

Figure 2. Surface roughness map created from standard deviation filtering of liDAR DeM with the use of $3 \times 3$ window size.

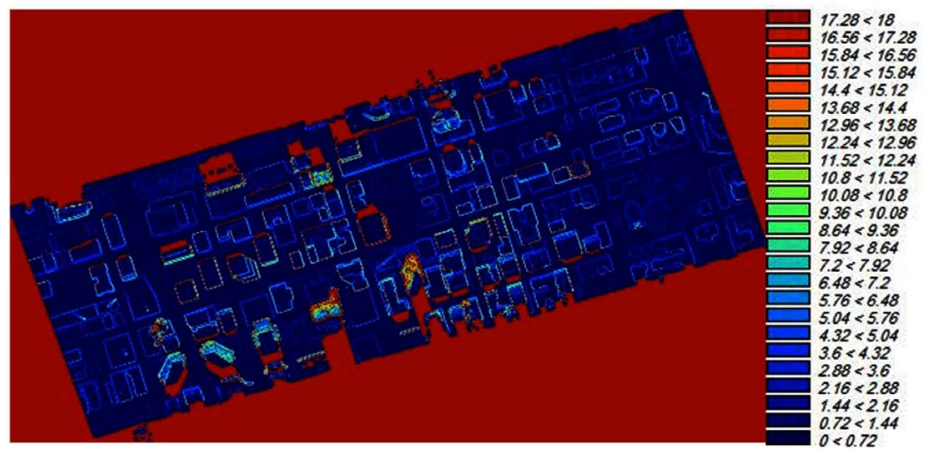

Figure 3. Surface roughness map created from standard deviation filtering of LiDAR DEM with the use of $5 \times 5$ window size. 


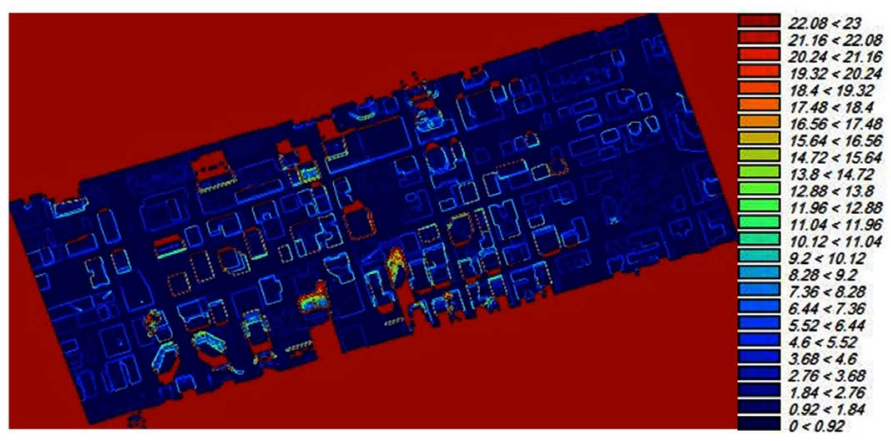

Figure 4. Surface roughness map created from standard deviation filtering of LiDAR DEM with the use of $7 \times 7$ window size.

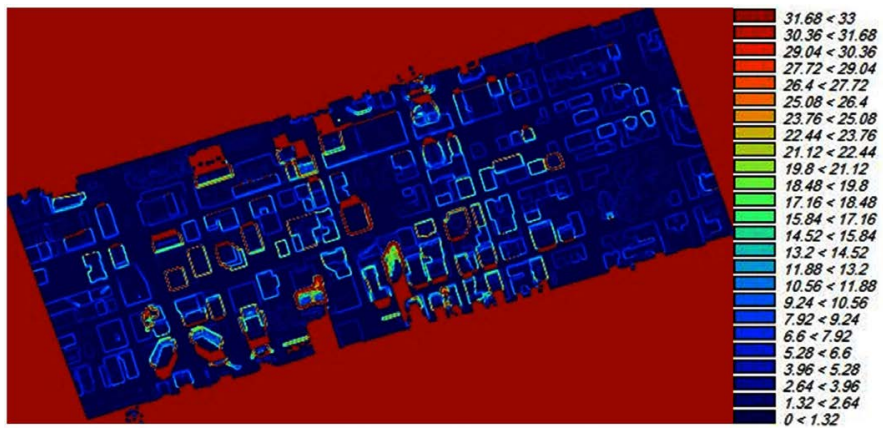

Figure 5. Surface roughness map created from standard deviation filtering of LiDAR DEM with the use of $11 \times 11$ window size.

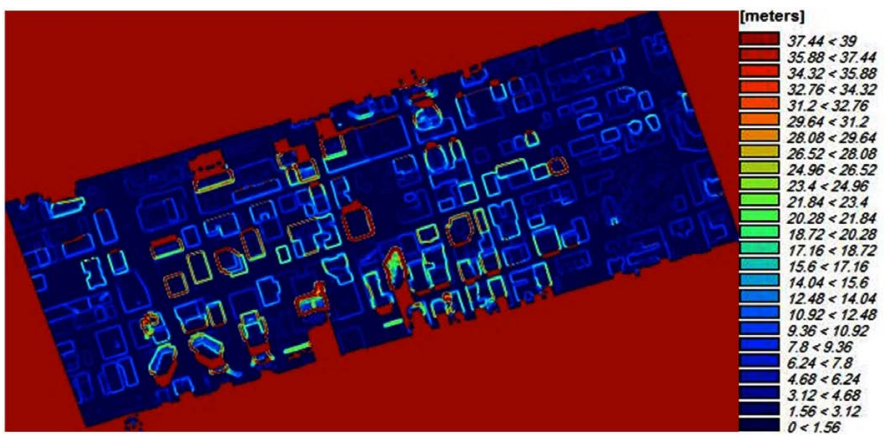

Figure 6. Surface roughness map created from standard deviation filtering of LiDAR DEM with ne use of $15 \times 15$ window size.

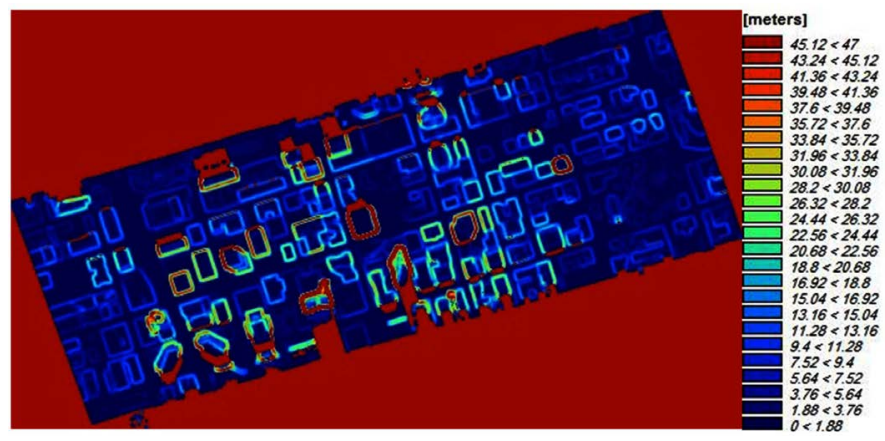

Figure 7. Surface roughness map created from standard deviation filtering of LiDAR DEM with the use of $21 \times 21$ window size 


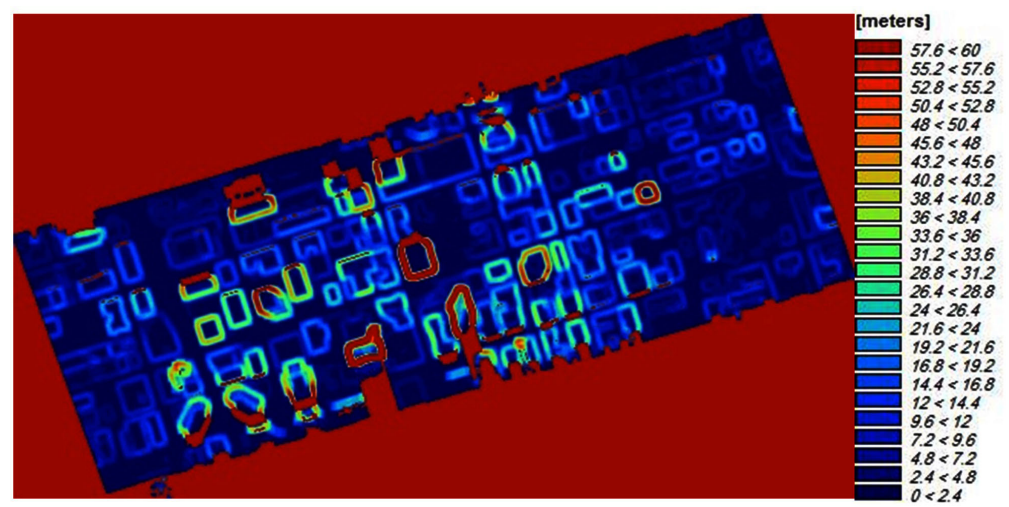

Figure 8. Surface roughness map created from standard deviation filtering of liDAR DEM with the use of $31 \times 31$ window size.

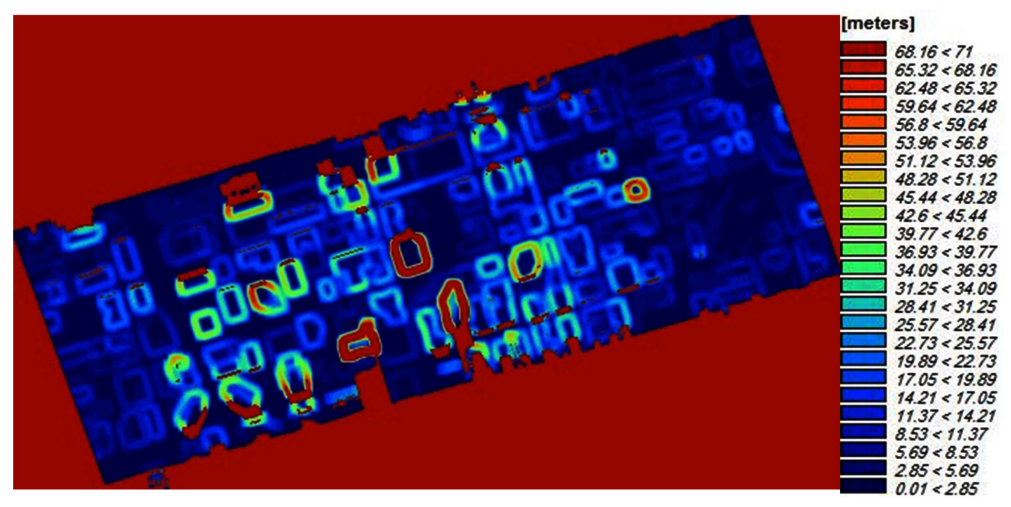

Figure 9. Surface roughness map created from standard deviation filtering of liDAR DEM with the use of $41 \times 41$ window size.

Table 1 depicts the results of the statistical analysis of the roughness maps created from standard deviation filtering of LiDAR DEM with varying window sizes. Also, Figure 10 depicts a chart that shows the effects of changing the window sizes of the standard deviation filter on the standard deviation, mean, median, root mean square and skewness of the created surface roughness map from standard deviation filtering of LiDAR DEM. Additionally, Figure 11 presents a chart showing the effects of changing the window size of the standard deviation filter on the ranges and kurtosis of the created surface roughness map from standard deviation filtering of LiDAR DEM. From Table 1 and Figure 10 it can be seen that each of the standard deviation (Stdev.), mean, median and Root Mean Square (RMS.) of the roughness maps increase with increasing the window sizes of the standard deviation filter, however the skewness which is a measure of symmetry of the normal distribution curve of the roughness map decreases with increasing the size of standard deviation filters which refers to that roughness maps created using higher window sizes are statistically more symmetrical compared to those created using smaller window size. Form Table 1 and Figure 11 the range of the surface roughness maps has increased by about $71.6 \%$ from the total increases due to increasing the size of the standard devia- 
tion filter from $3 \times 3$ to $5 \times 5$ while it decreases with increasing the window size more than $31 \times 31$. Also, Table 1 and Figure 11 depict the kurtosis which is a measure of whether the data are heavy-tailed or light-tailed relative to a normal distribution where datasets of high kurtosis tend to have heavy outliers while datasets of low kurtosis tend to lack outliers. From Table 1 and Figure 11 kurtosis decrease sharply with increasing the sizes of the filter window size till window size of $15 \times 15$ where the rate of decreasing is milder with bigger window sizes. From Table 1 and Figure 11 kurtosis has decreased by about 53\% due to increasing the window size from $3 \times 3$ to $7 \times 7$ and by about $82 \%$ due to increase in the filter window size from $3 \times 3$ to $15 \times 15$. The results of kurtosis indicate that the roughness map created from filter $41 \times 41$ contains the most consistent surface roughness map. Moreover, the results of the statistical analysis show that the sum of the surface roughness values in the maps increase with increasing the window size of the standard deviation filter for all the created surface roughness maps.

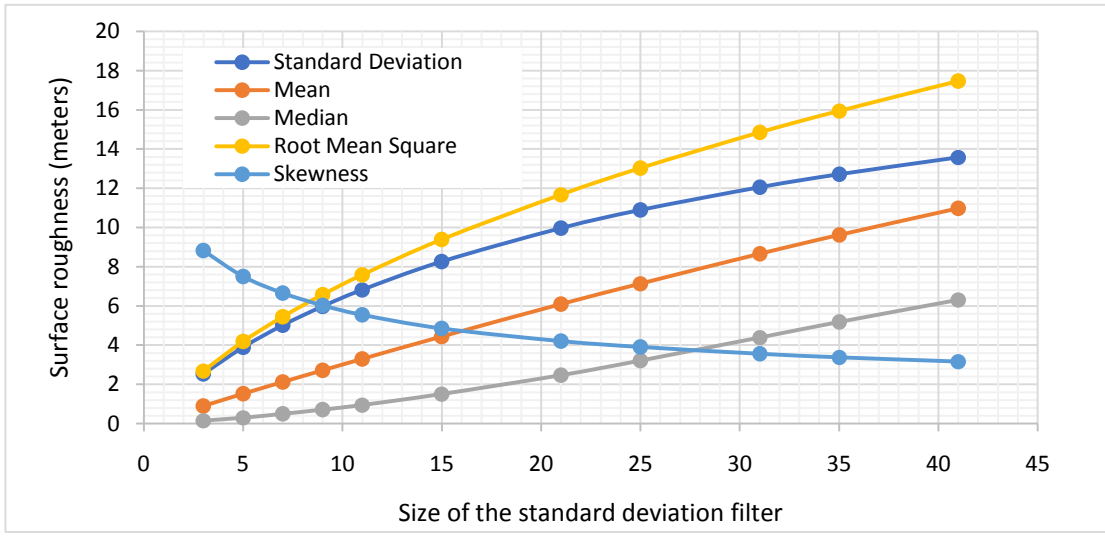

Figure 10. The effects of changing the window size of the standard deviation filter on the standard deviation mean, median root mean square and skewness of the created surface roughness map from standard deviation filtering ofLiDAR DEM.

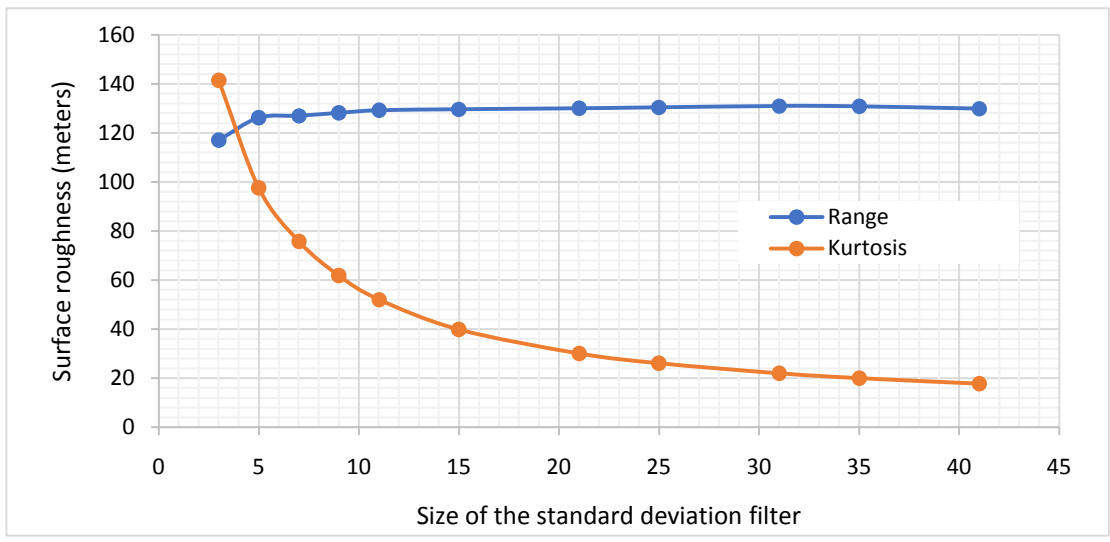

Figure 11. The effects of changing the window size of the standard deviation filter on the range and kurtosis of the created surface roughness map from standard deviation filtering of LiDAR DEM. 
Table 1. Statistical analysis results of the surface roughness maps from standard deviation filtering of LiDAR DEM at varying window sizes.

\begin{tabular}{|c|c|c|c|c|c|c|c|c|c|}
\hline Stdev. filter size & DEM stdev. & DEM stdev. & DEM stdev. & DEM stdev. & & & & DEM stdev & DEM stdev. \\
\hline Statist. quantity & filter $3 \times 3$ & filter $7 \times 7$ & filter $11 \times 11$ & filter $15 \times 15$ & filter $21 \times 21$ & filter $25 \times 25$ & filter $31 \times 31$ & filter $35 \times 35$ & filter $41 \times 41$ \\
\hline Min. (m) & 0 & 0 & 0 & 0 & 0 & 0.00065 & 0.000651 & 0.00973 & 0.01150 \\
\hline Max. (m) & 117.120 & 127.107 & 129.277 & 129.759 & 130.123 & 130.548 & 131.060 & 130.910 & 130.036 \\
\hline Mean (m) & 0.901 & 2.125 & 3.304 & 4.448 & 6.092 & 7.146 & 8.663 & 9.624 & 10.981 \\
\hline Median (m) & 0.160 & 0.496 & 0.9581 & 1.517 & 2.474 & 3.216 & 4.399 & 5.188 & 6.317 \\
\hline RMS(m) & 2.693 & 5.454 & 7.589 & 9.388 & 11.685 & 13.031 & 14.849 & 15.951 & 17.468 \\
\hline Stdev. (m) & 2.539 & 5.023 & 6.832 & 8.267 & 9.9716 & 10.896 & 12.060 & 12.721 & 13.586 \\
\hline Range (m) & 117.12 & 127.11 & 129.277 & 129.759 & 130.123 & 130.547 & 131.059 & 130.900 & 130.024 \\
\hline Skewness (m) & 8.822 & 6.6597 & 5.5461 & 4.8534 & 4.206 & 3.906 & 3.567 & 3.390 & 3.172 \\
\hline Kurtosis & 141.498 & 75.771 & 52.152 & 39.905 & 30.130 & 26.160 & 22.054 & 20.0759 & 17.805 \\
\hline Sum (m) & $2,420,492$ & $5,710,244$ & $8,879,719$ & $11,953,851$ & $16,372,277$ & $19,205,322$ & $23,281,319$ & $25,866,209$ & $29,512,019$ \\
\hline
\end{tabular}

\section{Creation and Analysis of Surface Roughness Maps from Standard Deviation Filtering of LiDAR Slope Gradient Model}

In this study surface roughness maps have been created from standard deviation filtering of a slope gradient model extracted from airborne LiDAR measurements where varying sizes of the standard deviation filter widow as $3 \times 3,5 \times 5$, $7 \times 7,9 \times 9,11 \times 11,15 \times 5,21 \times 21,25 \times 25,31 \times 31,35 \times 35$ and $41 \times 41$ have been used. Figures 12-19 represent eight of the created surface roughness maps with the use of the standard deviation filtering of varying window sizes of $3 \times 3$, $5 \times 5,7 \times 7,11 \times 11,15 \times 5,21 \times 21,31 \times 31$ and $41 \times 41$ respectively. In Figure 12 bright blue color tones dominate the roughness map from standard deviation filtering of the slope model using window size of $3 \times 3$ referring to roughness of values located at the middle of the range of the legend. However, the main streets are represented in dark blue colors referring to low roughness while the building blocks are represented in bright blow color tones referring high roughness values. The roughness map, Figure 12 is generally structured of high values of roughness with rough texture representing high roughness values due to big varieties of features of different geometrical dimensions. Figure 13 depicts a roughness map created from standard deviation filtering of the same slope map but with the use of window size of $5 \times 5$. Brighter blue color tones dominate the roughness map, Figure 13 compared to that in Figure 12 where bigger patches of similar color tones and rougher textures with corresponding higher roughness values and higher ranges of roughness values in the legend. With increasing the widow size of the standard deviation filter higher roughness values are represented in the roughness maps obtained using window sizes of $7 \times$ 7 and $11 \times 11$ that depicted in Figure 14 and Figure 15 respectively. In Figure 14 and Figure 15 amounts of bright blue color tones have increased with de- 
creasing in the areas of dark blue color tones of low roughness values in addition to appearance of yellow color tones at the rooftops of the buildings referring to high roughness values and rougher textures dominating the roughness maps. With more increasing in the window sizes of the standard deviation filter to $15 \times$ 15 and $21 \times 21$ more structured roughness maps have been obtained as depicted in Figure 16 and Figure 17 respectively where the bright blue color tones have increased with the increasing of the yellow to orange color tones in addition to rougher textures expressing wide variations in the color tones dominate the roughness map. Figure 18 and Figure 19 depict the most structured roughness maps with big variations in the color tones and rough textures reflecting big variations in the roughness values reflecting the wide varieties in the downtown landscape. Also, Figure 18 and Figure 19 show wide changes in the color tones staring from the dark blue color tones of low roughness values to bright blue color tones to yellow color tones to orange color tones and finally to red color tones of high roughness values. This refers to very rough texture of the roughness maps created with the use of standard deviation filters with window sizes of $31 \times 31$ and $41 \times 41$.

The statistical analysis results of the roughness maps created from standard deviation filtering of the slope gradient model extracted from LiDAR measurements with varying window sizes are shown in Table 2. Also, Figure 20 represents a chart that shows the effects of changing the window size of the standard deviation filter on the standard deviation, mean, median, root mean square and range of the created surface roughness map. Additionally, Figure 21 presents a chart that shows the effects of changing the window size of the standard deviation filter on the skewness and kurtosis of the created surface roughness map. From Table 2 and Figure 20 it can be seen that the mean, median and root mean square of the roughness maps increase due to increasing the window size of the standard deviation filter with relatively high rate till window size of 15 $\times 15$ where the rate of increase in these quantities become smaller with greater window sizes of the standard deviation filter. Alternatively, from Table 2 and Figure 20 the range of roughness maps decreases gradually with decreases in the window size of the standard deviation filter. On the other hand, the standard deviation of the roughness map increases due to increasing the window size of the of the standard deviation filter till window size of $15 \times 15$ where more increases in the window size of the standard deviation filter results in decreases in the standard deviation of the roughness map. From Table 2 and Figure 21 it can be seen that the skewness decreases in value with increasing the window size of standard deviation filter which refers to that roughness maps created standard deviation filter of high window sizes are of more symmetrical normal distribution compared to those created using standard deviation filter of small window sizes. In this context, the skewness decreases with higher rate till filter of $15 \times 15$ window size compared to the rate with more increasing in the size of the window of the standard deviation filter. Also, from Table 2 and Figure 21 kurtosis decreases sharply with increasing the window sizes of the filter till window size 
of $11 \times 11$ then it starts to increase with bigger window sizes of the standard deviation filter indicating that the roughness map created from filter $11 \times 11$ contains the most of consistent surface roughness values. Furthermore, Table 2 shows that sum of the surface roughness values in the maps increase with increasing the window size of the standard deviation filter for all the created surface roughness maps from standard deviation filtering of the LiDAR slope gradient model.

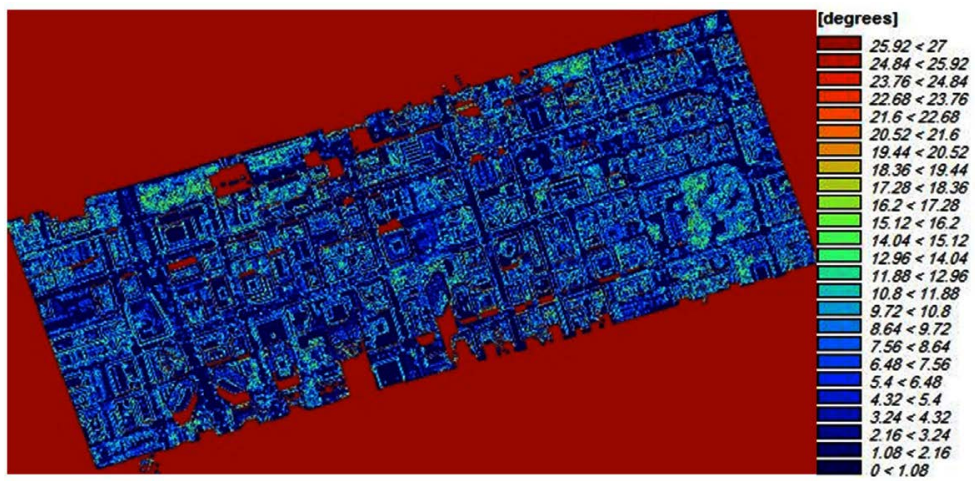

Figure 12. Surface roughness map created from standard deviation filtering of liDAR slope gradient model with the use of $3 \times 3$ window size.

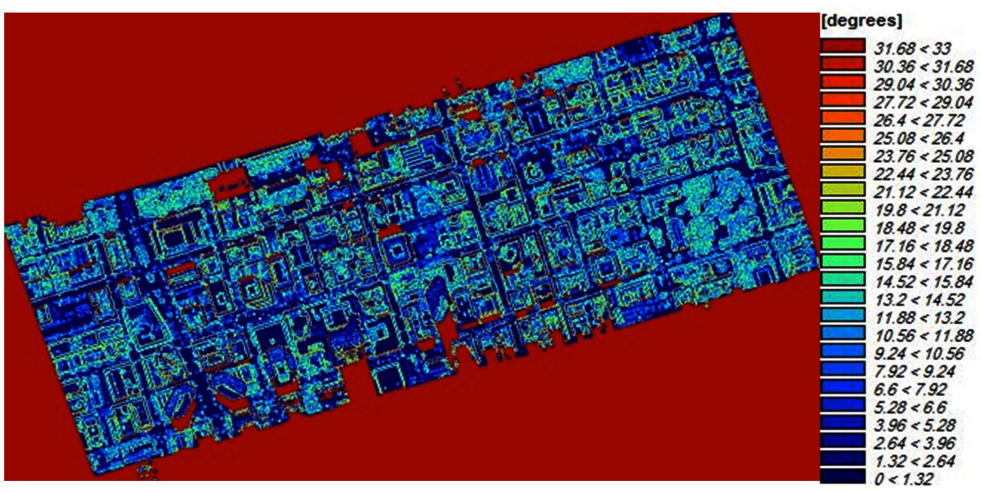

Figure 13. Surface roughness map created from standard deviation filtering of liDAR slope gradient model with the use of $5 \times 5$ window size.

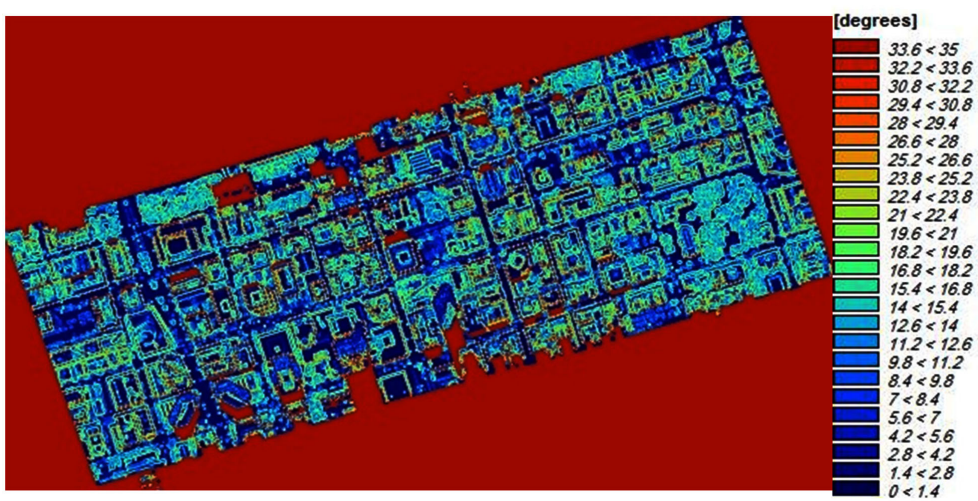

Figure 14. Surface roughness map created from standard deviation filtering of lidar slope gradient model with the use of $7 \times 7$ window size. 


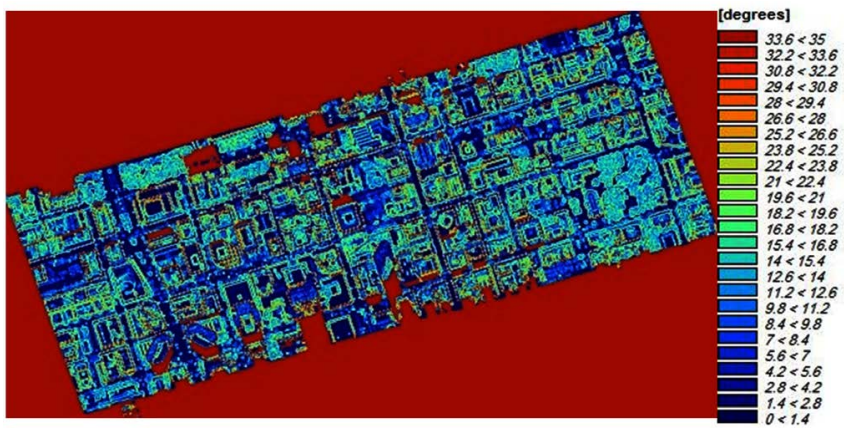

Figure 15. Surface roughness map created from standard deviation filtering of lidar slope gradient model with the use of 1111 window size.

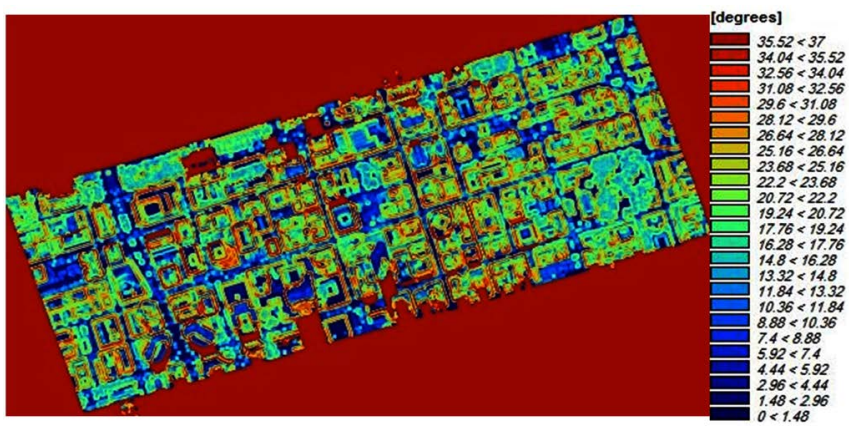

Figure 16. Surface roughness map created from standard deviation filtering of liDaR slope gradient model with the use of $15 \times 15$ window size.

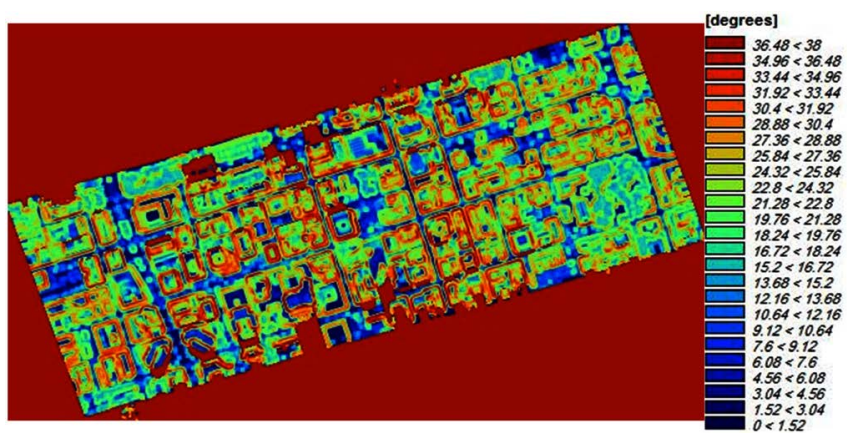

Figure 17. Surface roughness map created from standard deviation filtering of liDAR slope gradient model with the use of $21 \times 21$ window size.

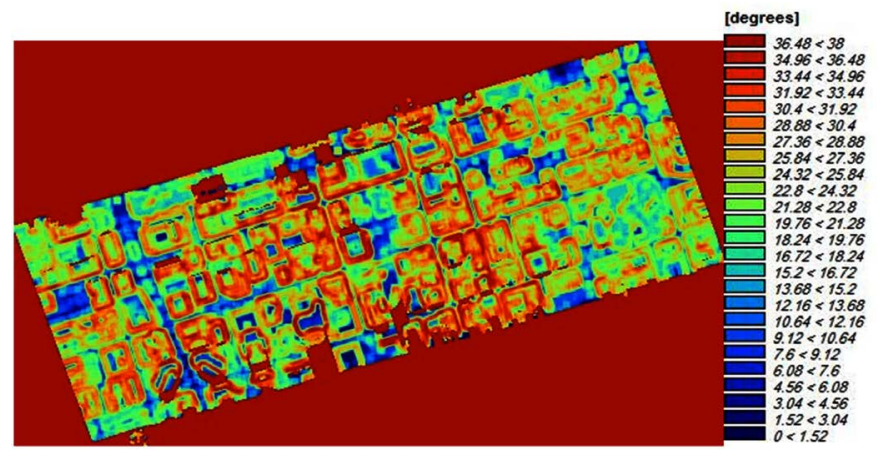

Figure 18. Surface roughness map created from standard deviation filtering ofliDaR slope gradient model with the use of $31 \times 31$ window size. 


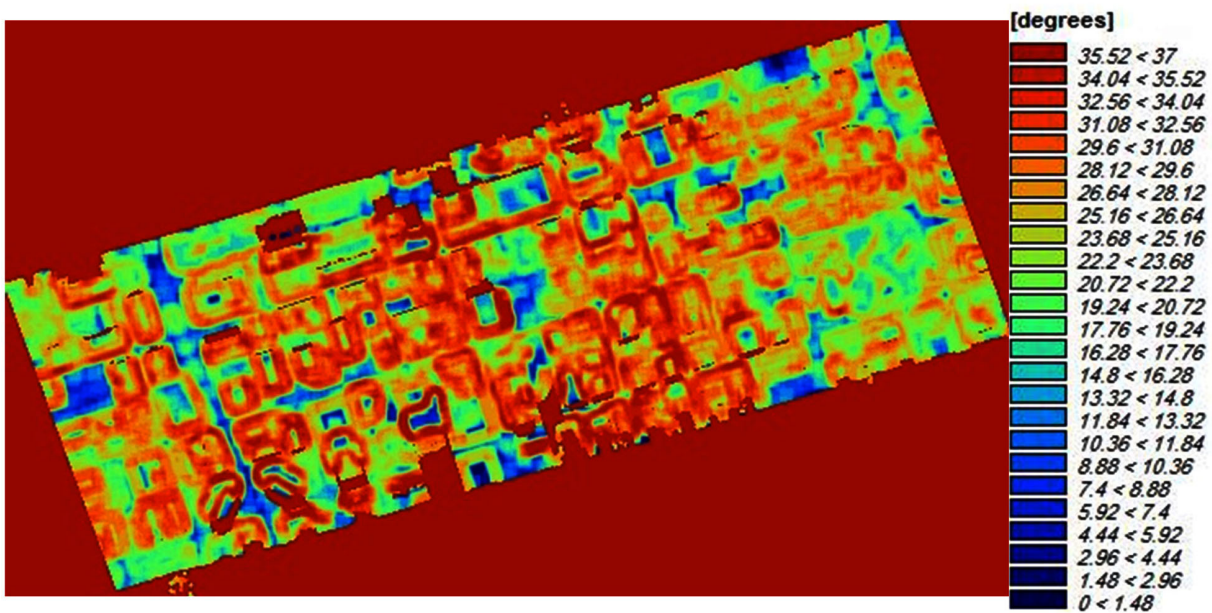

Figure 19. Surface roughness map created from tandard deviation filtering of liDaR slope gradient model with the use of $41 \times 41$ window size.

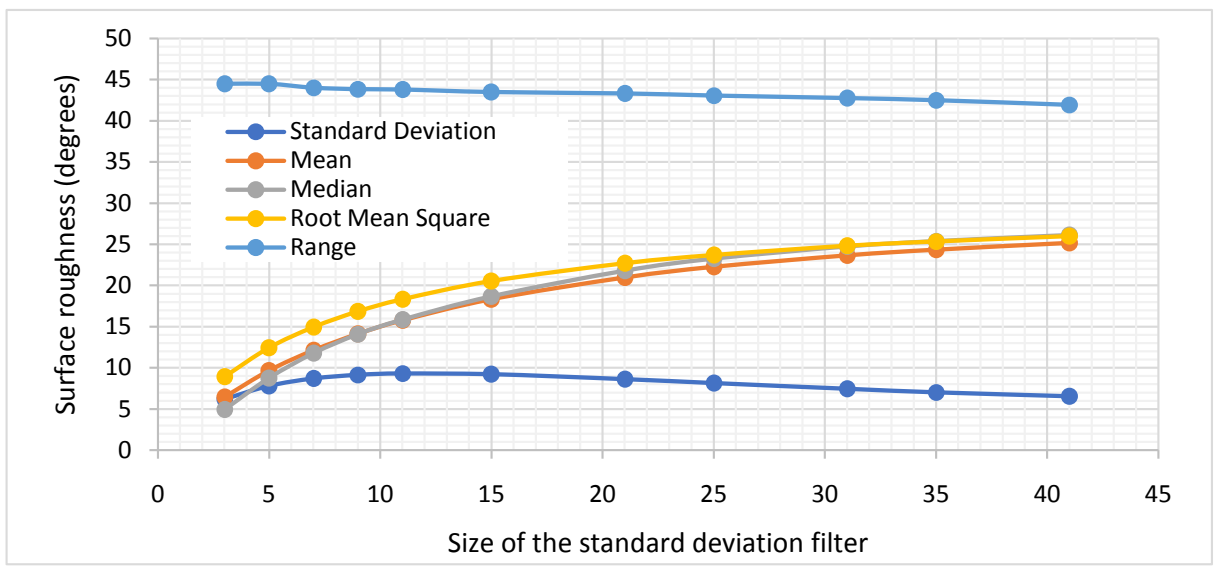

Figure 20. The effects of changing the size of the standard deviation filter on the standard deviation the mean, the median, root mean square and the range of the created surface roughness map from standard deviation filtering of the slope gradient model.

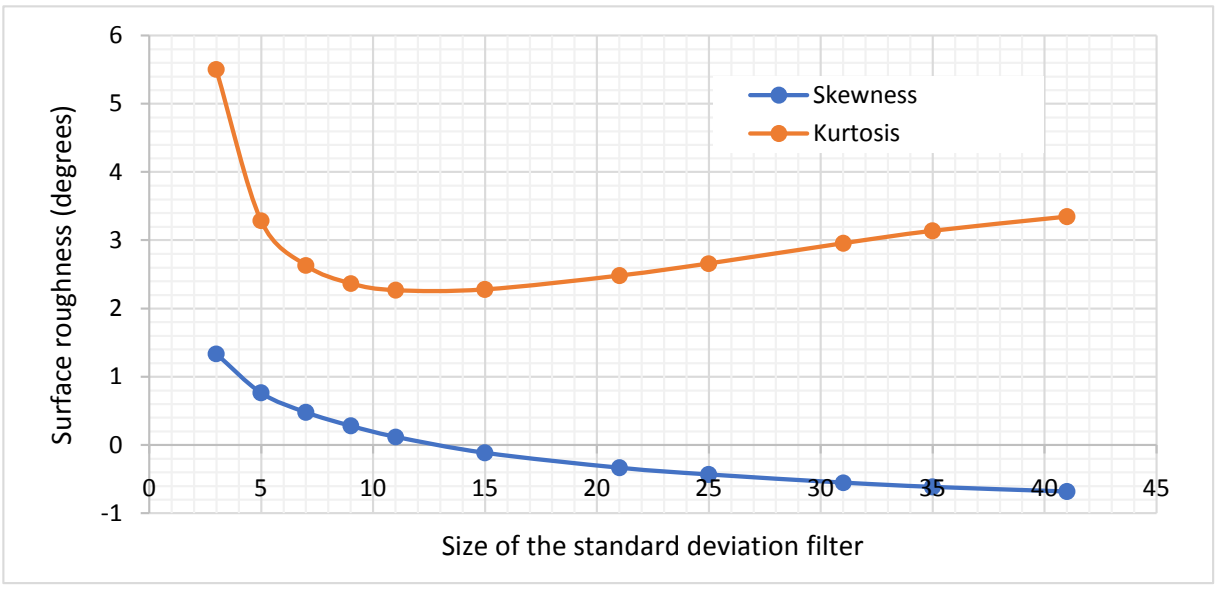

Figure 21. The effects of changing the size of the standard deviation filter on the skewness and kurtosis of the created surface roughness map from standard deviation filtering of the slope gradient model. 
Table 2. Statistical analysis results of surface roughness maps obtained from standard deviation filtering of LiDAR slope gradient model at varying window sizes.

\begin{tabular}{|c|c|c|c|c|c|c|c|c|c|}
\hline Stdev. filter size & \multirow{2}{*}{$\begin{array}{l}\text { Slope stdev } \\
\text { filter } 3 \times 3\end{array}$} & \multirow{2}{*}{$\begin{array}{l}\text { Slope stdev } \\
\text { filter } 5 \times 5\end{array}$} & \multirow{2}{*}{$\begin{array}{l}\text { Slope stdev } \\
\text { filter } 9 \times 9\end{array}$} & \multirow{2}{*}{$\begin{array}{l}\text { Slope stdev } \\
\text { filter } 15 \times 15\end{array}$} & \multirow{2}{*}{$\begin{array}{r}\text { Slope stdev } \\
\text { filter } 21 \times 21\end{array}$} & \multirow{2}{*}{$\begin{array}{l}\text { Slope stdev } \\
\text { filter } 25 \times 25\end{array}$} & \multirow{2}{*}{$\begin{array}{c}\text { Slope stdev } \\
\text { filter } 31 \times 31\end{array}$} & \multirow{2}{*}{$\begin{array}{l}\text { Slope stdev } \\
\text { filter } 35 \times 35\end{array}$} & \multirow{2}{*}{$\begin{array}{l}\text { Slope stdev } \\
\text { filter } 41 \times 41\end{array}$} \\
\hline Statist. quantity & & & & & & & & & \\
\hline Min. (deg.) & 0 & 0 & 0 & 0 & 0 & 0.114319 & 0.11432 & 0.11432 & 0.34275 \\
\hline $\operatorname{Max}($ deg.). & 44.478 & 44.464 & 43.846 & 43.468 & 43.307 & 43.177 & 42.856 & 42.616 & 42.286 \\
\hline Mean (deg.) & 6.450 & 9.666 & 14.125 & 18.3424 & 20.987 & 22.255 & 23.666 & 24.363 & 25.154 \\
\hline Median (deg.) & 4.935 & 8.759 & 14.103 & 18.680 & 21.809 & 23.246 & 24.741 & 25.399 & 26.102 \\
\hline RMS. (deg.) & 8.937 & 12.437 & 16.84 & 20.532 & 22.694 & 23.699 & 24.809 & 25.360 & 25.990 \\
\hline Stdev. (deg.) & 6.186 & 7.826 & 9.169 & 9.226 & 8.634 & 8.146 & 7.445 & 7.0419 & 6.542 \\
\hline Range (deg.) & 44.478 & 44.464 & 43.846 & 43.468 & 43.307 & 43.062 & 42.742 & 42.502 & 41.943 \\
\hline Skewness (deg.) & 1.340 & 0.765 & 0.280 & -0.115 & -0.334 & -0.431 & -0.549 & -0.614 & -0.683 \\
\hline Kurtosis (deg.) & 5.509 & 3.292 & 2.371 & 2.279 & 2.481 & 2.662 & 2.958 & 3.138 & 3.348 \\
\hline Sum (deg.) & $17,335,550$ & $25,977,889$ & $37,960,617$ & $49,296,268$ & $56,403,673$ & $59,811,826$ & $63,602,679$ & $65,477,095$ & $67,601,670$ \\
\hline
\end{tabular}

\section{Creation and Analysis of Surface Roughness Maps from Standard Deviation Filtering of LiDAR Profile Curvature Model}

Figures 22-29 represent the surface roughness maps created as results of standard deviation filtering of the profile curvature model extracted from Airborne LiDAR measurements using filter of varied window sizes as $3 \times 3,5 \times 5,7 \times 7,11$ $\times 11,15 \times 15,21 \times 21,31 \times 31$ and $41 \times 41$ respectively. In Figure 22 and Figure 23 dark blue color tones dominate the roughness map from standard deviation filter of window size of $3 \times 3$ referring to small roughness dominate the roughness map despite the existence of downtown landscape with high intensity of varied height features which refers to neglection of roughness due to important features in the area. Different from the roughness maps created from standard deviation filtering of the DEM and the slope gradient model the edges of the buildings are not clear with only main roads that can be interpretable from darker blue color tones. Also, the texture of the roughness map in Figure 22 and Figure 23 is relatively smooth at most parts of the roughness map where smoothing of roughness due to features at wide parts of the roughness map is very clear. Figure 24 and Figure 25 represent surface roughness maps created with standard deviation filter of window sizes of $7 \times 7$ and $11 \times 11$ depict brighter color tones compared to Figure 22 and Figure 23 referring to higher surface roughness values. With increasing the sizes of the standard deviation filters to 15 $\times 15$ and $21 \times 21$ more brighter roughness maps and represented in Figure 26 and Figure 27 have been obtained but blurred roughness maps have been the case with areas of different color patches dominate the roughness maps referring to rougher texture. In Figure 28 and Figure 29 which depict roughness maps from standard deviation filtering of the profile curvature model with window sizes of $31 \times 31$ and $41 \times 41$ much brighter color tone roughness maps have been 
obtained. However, much blurred maps without distinguishable features and increasing in the sizes of the color patches leading to wider changes in the color tones and rougher textures maps have been obtained.

The statistical analysis results of the roughness maps created from standard deviation filtering of the profile curvature model extracted from LiDAR measurements with the use of varying filter window sizes are shown in Table 3. Also, Figure 30 represents a chart that shows the effects of changing the size of the standard deviation filter window on the mean, median, root mean square and standard deviation of the created surface roughness map from standard deviation filtering of the profile curvature model. Moreover, Figure 31 presents a chart that represents the effects of changing the size of the standard deviation filter window on the range and skewness of the created surface roughness map from standard deviation filtering of the profile curvature model. From Table 3 and Figure 30 it can be seen that the mean, median and root mean square of the roughness map increase due to increasing the window size of the standard deviation filter with relatively high rate till filter size of $15 \times 15$ where the rate of increase become smaller due to bigger filter window sizes. On the other hand, the standard deviation of the roughness map decreases gradually due to increasing the window size of the of the standard deviation filter over all the tested window sizes. From Table 3 and Figure 31 the ranges and skewness of the roughness maps decrease with high rates due to increases in the window size of the standard deviation filter till filter size of about $15 \times 15$ where the rate of decreases become smaller with more increases in the filter window size. In this context the ranges of roughness decrease by about $93.6 \%$ of the total decrease while the skewness decreases by about $82.6 \%$ due to increasing the filter window size to 15 $\times 15$. Also, from Table 3 the kurtosis decreases with high rates due to increasing the size of the filter window where window size of $5 \times 5$ produces a decrease in kurtosis of about $58.6 \%$ while increasing the window size to $7 \times 7$ produce a decrease in kurtosis by about $76.3 \%$ and increasing the window size to $15 \times 15$ produce decreases in the kurtosis by about $93.76 \%$ of the total decrease in kurtosis which is due to increase in the window size to $41 \times 41$. On the other hand, Table 3 shows that sum of the surface roughness values in the maps increase gradually with increasing of the window sizes of the standard deviation filter for all the created surface roughness maps from standard deviation filtering of the profile curvature model.

\section{Discussions}

Three measures of the Earth's surface roughness have been tested for creation and analysis of surface roughness maps from airborne LiDAR measurements. A group of surface roughness maps have been created from standard deviation filtering of LiDAR DEM with varying filter window sizes. Also, another group of surface roughness maps have been obtained from standard deviation filtering of LiDAR slope gradient model with varying filter window sizes. Additionally, a 


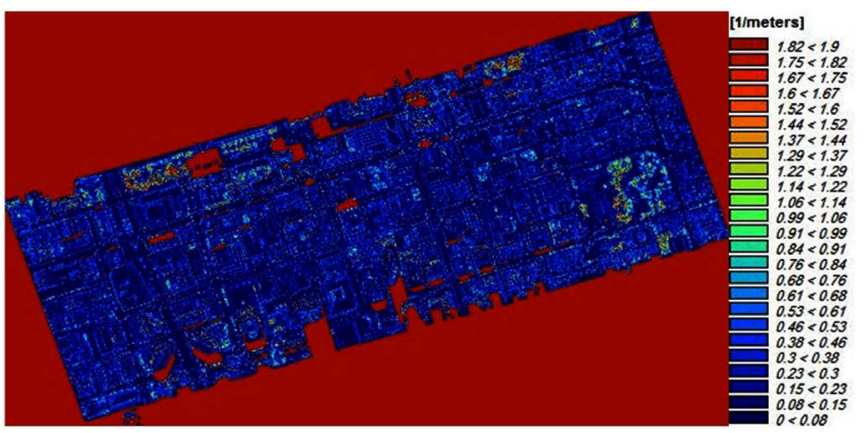

Figure 22. Surface roughness map created from standard deviation filtering of a profile curvature model with the use of window size of $3 \times 3$.

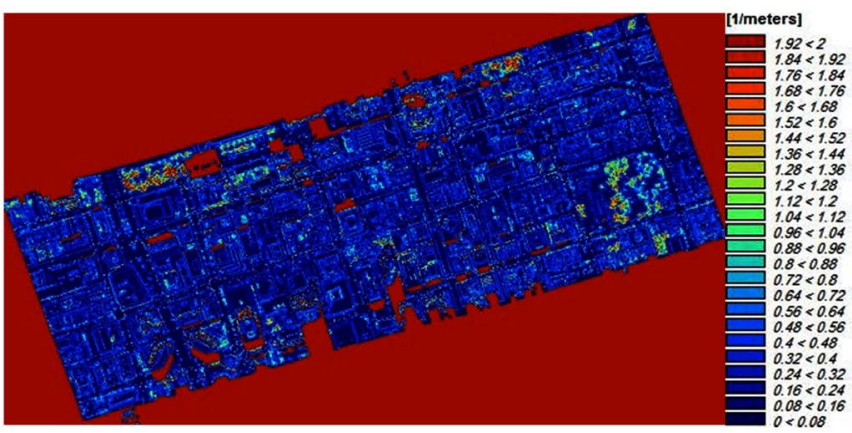

Figure 23. Surface roughness map created from standard deviation filtering of a profile curvature model with the use of window size of $5 \times 5$.

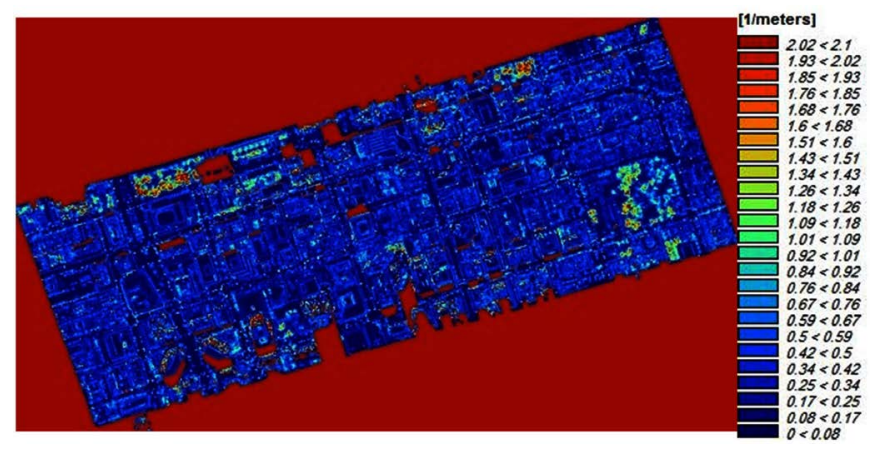

Figure 24. Surface roughness map created from standard deviation filtering of a profile curvature model with the use of window size of $7 \times 7$.

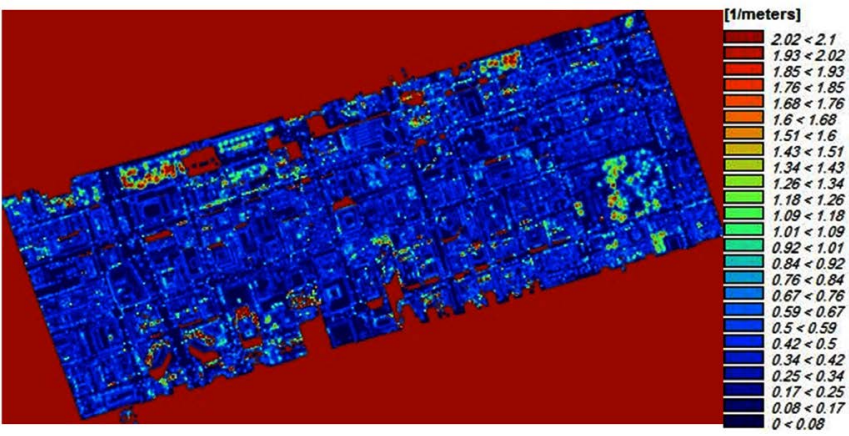

Figure 25 Surface roughness map created from standard deviation filtering of a profile curvature model with the use of window size of $11 \times 11$. 


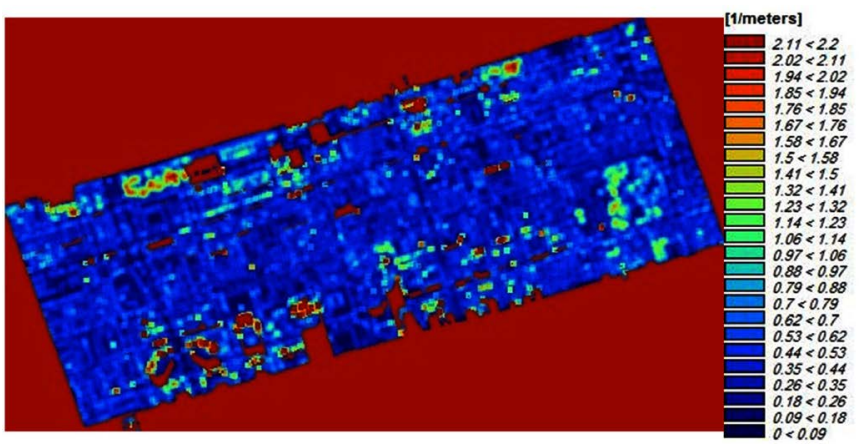

Figure 26. Surface roughness map created from standard deviation filtering of a profile curvature model with the use of window size of $15 \times 15$.

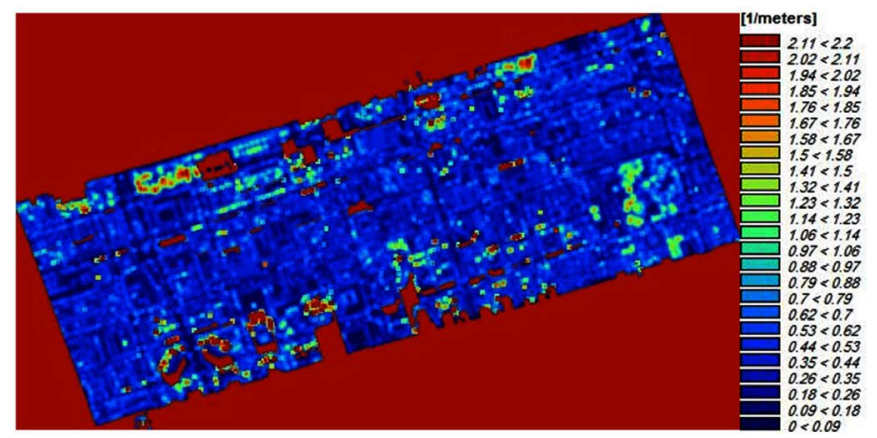

Figure 27. Surface roughness map created from standard deviation filtering of a profile curvature model with the use of window size of $21 \times 21$.

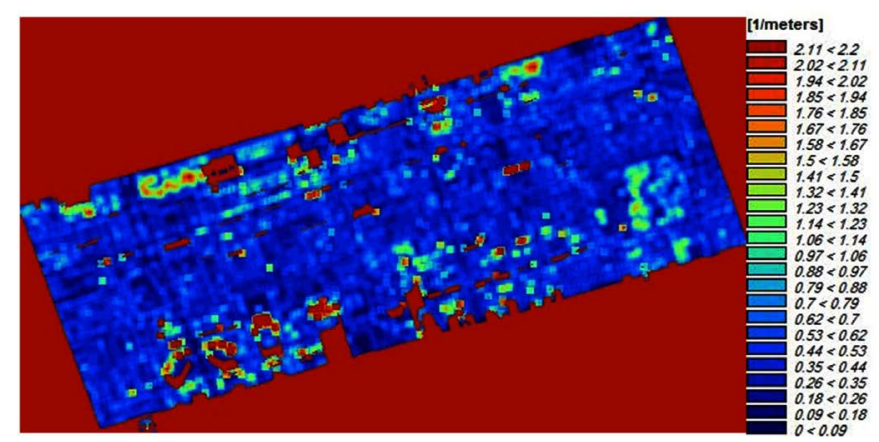

Figure 28. Surface roughness map created from standard deviation filtering of a profile curvature model with the use of window size of $31 \times 31$.

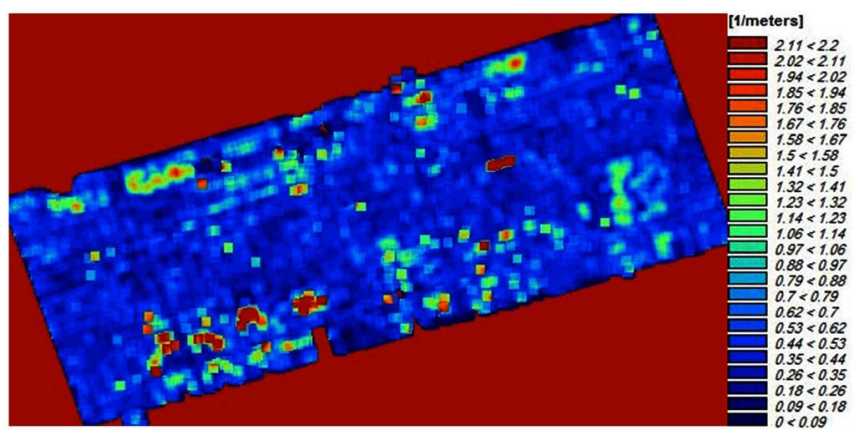

Figure 29. Surface roughness map created from standard deviation filtering of a profile curvature model with the use of window size of $41 \times 41$. 


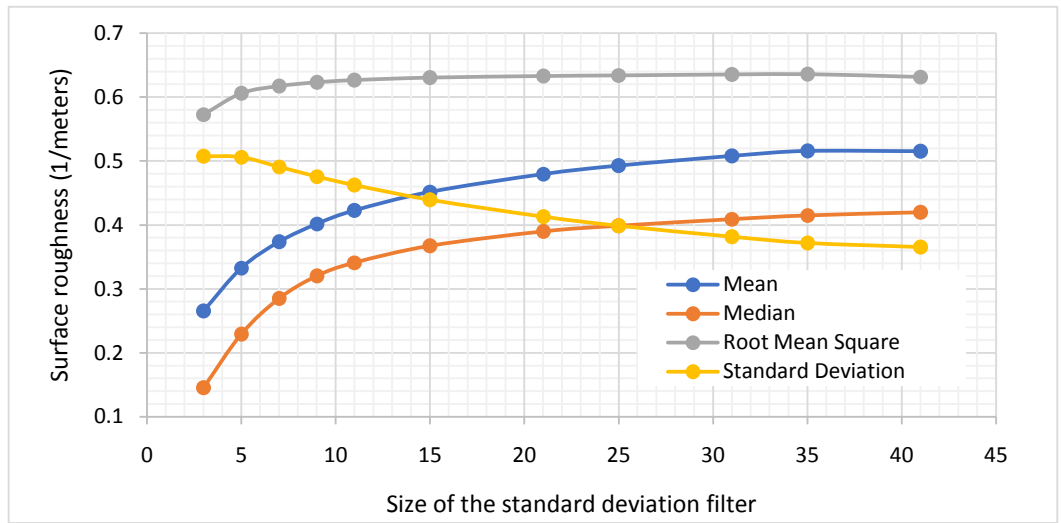

Figure 30. The effects of changing the window size of the standard deviation filter on the mean median root mean square and standard deviation of the created surface roughness maps from standard deviation filtering of the profile curvature model.

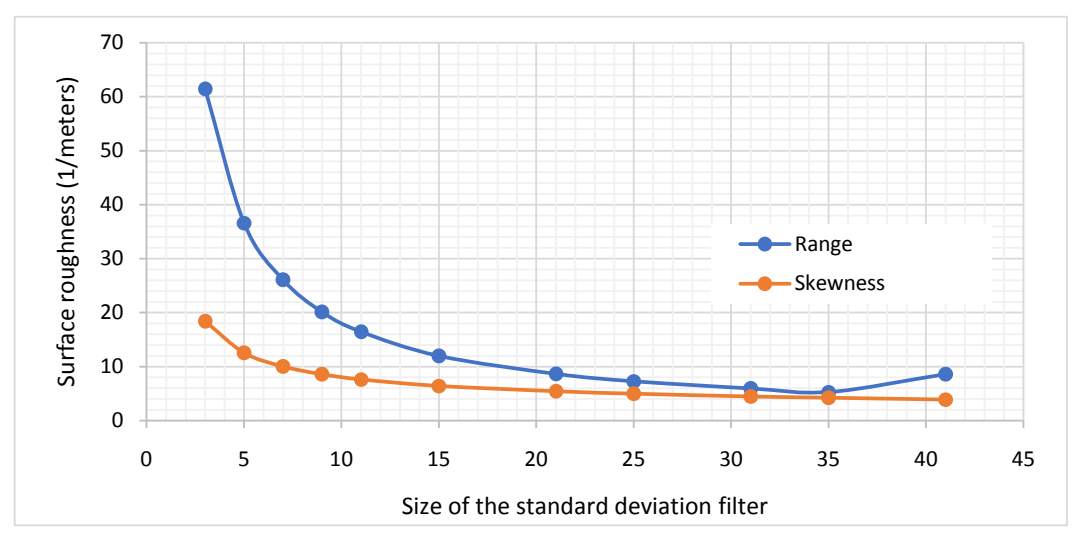

Figure 31. The effects of changing the window size of the standard deviation filter on the range and skewness of the created surface roughness maps from standard deviation filtering of the profile curvature model.

Table 3. Statistical analysis results of the surface roughness maps obtained from standard deviation filtering of the profile curvature model created from airborne LiDAR measurements at varying sizes of the filter window.

\begin{tabular}{|c|c|c|c|c|c|c|c|c|c|}
\hline $\begin{array}{l}\text { Stdev. filter size } \\
\text { Statist. quantity }\end{array}$ & $\begin{array}{c}\text { Profile } \\
\text { curvat. } \\
\text { filter } 3 \times 3\end{array}$ & $\begin{array}{c}\text { Profile } \\
\text { curvat. } \\
\text { filter } 5 \times 5\end{array}$ & $\begin{array}{c}\text { Profile } \\
\text { curvat. filter } \\
7 \times 7\end{array}$ & $\begin{array}{c}\text { Profile } \\
\text { curvat. filter } \\
9 \times 9\end{array}$ & $\begin{array}{c}\text { Profile } \\
\text { curvat. filter } \\
11 \times 11\end{array}$ & $\begin{array}{c}\text { Profile } \\
\text { curvat. filter } \\
15 \times 15\end{array}$ & $\begin{array}{c}\text { Profile } \\
\text { curvat. filter } \\
25 \times 25\end{array}$ & $\begin{array}{c}\text { Profile } \\
\text { curvat. filter } \\
35 \times 35\end{array}$ & $\begin{array}{c}\text { Profile } \\
\text { curvat. filter } \\
41 \times 41\end{array}$ \\
\hline Min. $\left(\mathrm{m}^{-1}\right)$ & 0 & 0 & 0 & 0 & 0 & 0 & 0 & 0.0031 & 0 \\
\hline $\operatorname{Max} .\left(m^{-1}\right)$ & 61.434 & 36.631 & 26.120 & 20.137 & 16.467 & 12.010 & 7.324 & 5.322 & 8.623 \\
\hline $\operatorname{Mean}\left(\mathrm{m}^{-1}\right)$ & 0.266 & 0.333 & 0.374 & 0.402 & 0.423 & 0.452 & 0.493 & 0.516 & 0.515 \\
\hline $\operatorname{Median}\left(\mathrm{m}^{-1}\right)$ & 0.146 & 0.229 & 0.285 & 0.320 & 0.341 & 0.367 & 0.399 & 0.415 & 0.420 \\
\hline RMS. $\left(\mathrm{m}^{-1}\right)$ & 0.573 & 0.606 & 0.617 & 0.623 & 0.627 & 0.630 & 0.634 & 0.636 & 0.632 \\
\hline Stdev. $\left(m^{-1}\right)$ & 0.508 & 0.506 & 0.491 & 0.476 & 0.462 & 0.439 & 0.399 & 0.372 & 0.365 \\
\hline Range $\left(m^{-1}\right)$ & 61.434 & 36.631 & 26.120 & 20.137 & 16.470 & 12.010 & 7.324 & 5.319 & 8.623 \\
\hline Skewness $\left(\mathrm{m}^{-1}\right)$ & 18.424 & 12.569 & 10.040 & 8.608 & 7.663 & 6.457 & 4.984 & 4.248 & 3.934 \\
\hline Kurtosis $\left(\mathrm{m}^{-1}\right)$ & 938.40 & 405.66 & 244.52 & 171.44 & 130.38 & 86.86 & 46.666 & 32.234 & 29.100 \\
\hline $\operatorname{Sum}\left(\mathrm{m}^{-1}\right)$ & 713,782 & 894,517 & $1,004,256$ & $1,080,285$ & $1,136,434$ & $1,214,443$ & $1,324,695$ & $1,386,030$ & $1,549,376$ \\
\hline
\end{tabular}


third group of surface roughness maps have been generated from standard deviation filtering of LiDAR profile curvature model with varying filter window sizes. The three measures have been examined against the window size of the standard deviation filter in downtown urban landscape characterized by high intensity of varied geometry, sizes, shapes, heights, and types of features.

Visual analysis of the surface roughness maps from standard deviation filtering of LiDAR DEM has shown dark blue color tones dominate the roughness map from window size of $3 \times 3$ referring to small roughness values. Small changes have occurred on the roughness maps from window size of $5 \times 5$ where brighter color tone roughness map has been obtained but high degree of feature smoothing is still there. However, more increases in the window sizes of the standard deviation filters has given more structured roughness maps of rougher textures. The roughness map created with the use of window sizes of $31 \times 31$ and $41 \times 41$ have been the most structured roughness maps with relatively wide variation of the color tones within the maps and rough texture. Statistical analysis of the roughness maps generated from standard deviation filtering of LiDAR DEM has indicated that the standard deviation, mean, median and root mean square of the surface roughness maps have increased with increasing the window size of the standard deviation filter while the skewness has decreased with increasing the window size of standard deviation filter indicating that larger window sizes have produced statistically more symmetrically surface roughness maps. Additionally, the ranges of the surface roughness have increased by about $71.6 \%$ with changing the window size from $3 \times 3$ to $5 \times 5$, however it decreases with window sizes greater than $31 \times 31$. Moreover, the kurtosis has decreased sharply with increasing the window size till $15 \times 15$ while the rate of decreasing has become milder with more increases. That is kurtosis has decreased by about $53 \%$ and $82 \%$ due to increasing the window size to $7 \times 7$ and $15 \times 15$ respectively.

The surface roughness maps from standard deviation filtering of LiDAR slope gradient model have showed differences compared to their corresponding from filtering of LiDAR DEM where brighter blue color tones dominate the roughness map from window size of $3 \times 3$ referring to roughness values at the middle of the legend. The main streets have been represented in low roughness values of dark blue color tones as expected while the blocks of buildings have given roughness of bright blue color tones producing rough texture map. Similar to the roughness maps from LiDAR DEM, with increasing the window sizes of the standard deviation filters more structured roughness maps have been obtained with increases in the brighter blue tones and rougher textures. The roughness maps from window sizes of $31 \times 31$ and $41 \times 41$ have showed wide changes in the color tones producing rough texture roughness maps. Again, the statistical analysis of the LiDAR slope gradient roughness maps has indicated that the mean, median and root mean square of the roughness maps have increased due to increasing the size of the standard deviation filter with higher rates till $15 \times 15$ while the ranges of the roughness maps have decreased gradually with the in- 
creases in the window size. Also, the standard deviation of the roughness map has increased due to increasing the window size till $15 \times 15$ but more increases in the window size have given decreases in the standard deviation of the roughness map. Moreover, the skewness has decreased due to increasing the window size till $15 \times 15$ while it has increased with more increases indicating that the roughness maps from window size of $15 \times 15$ is the most symmetrical one. Furthermore, the kurtosis has decreased with relatively high rate due to increasing the filter window size till $11 \times 11$ where milder rates of decreases have occurred with larger window sizes indicating that the roughness map from window size of $11 \times 11$ is the most consistent.

Visual analysis of the standard deviation filtering of profile curvature model has indicated that dark blue color tones of small roughness values dominate the roughness maps from window sizes of $3 \times 3$ and $5 \times 5$ with the edges of the building cannot be easily distinguishable. With increasing the filter window sizes to $15 \times 15$ and $21 \times 21$ brighter but burred roughness maps have been obtained. However, in the roughness maps from window sizes of $31 \times 31$ and $41 \times 41$ much brighter color tones dominate the roughness maps but much blurred maps with hardly distinguishable features. The statistical analysis results of the roughness maps from standard deviation filtering of the profile curvature model have not been much different from those discussed earlier. The mean, median and root mean square of the roughness maps have increased with high rates due to increasing the filter window sizes till $15 \times 15$ where the rate of increase become smaller with larger sizes however, the standard deviation of the roughness map has decreased gradually due to increasing the filter window size. On the other hand, the ranges and skewness of the roughness maps have decreased with high rates due to increases in the filter window sizes till $15 \times 15$ while the rate of decreases become smaller with bigger window sizes. In this context, the ranges and skewness have decreased by about $93.6 \%$ and $82.6 \%$ respectively of the total decrease due to increasing the filter window size to $15 \times 15$. Also, the kurtosis has decreased with high rates due to increasing the filter window size where decreases in kurtosis of 58.6\%, 76.3\% and $93.76 \%$ have occurred due to increases in the window sizes to $5 \times 5,7 \times 7$ and $15 \times 15$ respectively.

\section{Conclusions}

The Earth's surface roughness constitutes an important parameter in terrain analysis for studying different environmental and engineering problems. Authors gave different definitions and measures for the earth's surface roughness that usually depend on exploitation of digital elevation data for its reliable determination. This research aimed at exploring the different approaches for defining and extraction of the Earth's surface roughness from Airborne LiDAR Measurements. It also aimed at evaluating the effects of the window size of the standard deviation filter on the created roughness maps in downtown landscapes using three different measures for surface roughness namely; standard deviation 
filtering of the DEM, standard deviation filtering of the slope gradient model and standard deviation filtering of the profile curvature model. A complete tile of Airborne LiDAR measurement for the downtown of the City of Toronto, Canada has been exploited in creation of three groups of surface roughness maps with the use of the above mentioned three measures at varying window sizes of the standard deviation filter. The created surface roughness maps have been analyzed visually and statistically against the window size of the standard deviation filter in downtown urban landscape characterized by high intensity of varied sizes, shapes, heights, and types of features. Visual analysis has shown dark blue color tones of small roughness values dominate the roughness map from standard deviation filtering of LiDAR DEM with window size of $3 \times 3$, however, with increasing the filter window size, brighter blue color tones of higher roughness values and thicker edges of the buildings with more structured roughness maps have been obtained. However, bright blue color tones dominate the surface roughness map from standard deviation filtering of LiDAR slope gradient model using $3 \times 3$ window size while the main streets have been represented in dark blue color tones. Also, increasing the window size of the filter has given structured roughness maps of higher roughness values and wider changes in the color tones starting from dark blue to bright blue to yellow to orange color tones and finally to red color tones of high roughness values producing rough texture roughness maps. In the case of the roughness maps from standard deviation filtering of LiDAR profile curvature model, dark blue color tones have dominated the roughness maps from window sizes of $3 \times 3$ and $5 \times 5$ indicating that small window sizes produce small roughness values with edges of buildings not being easily distinguishable. However, with increasing the window sizes, brighter but blurred roughness maps have been obtained with wide areas of different color patches giving rough texture maps.

Statistical analysis has provided more understanding of the outcomes from the visual analysis where in the roughness maps from LiDAR DEM, the standard deviation, mean, median, root mean square and range have increased with increasing the filter window size where the ranges have increased by $71.6 \%$ due to increasing the window size from $3 \times 3$ to $5 \times 5$. On the other hand, the skewness and kurtosis have decreased with increasing the window size of the filter indicating that roughness maps from larger window sizes are statistically more symmetrical and more consistent since kurtosis has decreased by about $53 \%$ and $82 \%$ due to increasing the window size to $7 \times 7$ and $15 \times 15$ respectively. Close results have been obtained from the analysis of the roughness maps created from slope gradient model where, the mean, median and root mean square of the roughness maps have increased with high rates due to increasing the filter window size till $15 \times 15$ while the range has decreased gradually with increasing the window size. Additionally, the standard deviation of the roughness map has increased with increasing the window size till $15 \times 15$ while it has decreased with more increases. However, the skewness analysis has shown that window size of 
$15 \times 15$ has given the most symmetrical roughness map while the kurtosis analysis has shown that window size of $11 \times 11$ has given the most consistent one. Moreover, the statistical analysis of the roughness maps from profile curvature model has indicated that the mean, median and root mean square of the roughness maps have increased due to increasing the window size with high rate till 15 $\times 15$ where the rate of increase become smaller with larger window sizes. However, the standard deviation, range, skewness and kurtosis of the roughness map have decreased due to increasing the window size of the filter where the range and skewness have decreased by $93.6 \%$ and $82.6 \%$ respectively due to increasing the window sizes to $15 \times 15$ while kurtosis has decreased by $58.6 \%, 76.3 \%$ and $93.76 \%$ due to window size increases to $5 \times 5,7 \times 7$ and $15 \times 15$ respectively. Exploitation of the roughness maps from the different approaches in real applications such as hydrodynamic modelling and flood studies could determine the efficiency of the different surface roughness measures in creation of reliable surface roughness maps in different landscapes.

\section{Acknowledgements}

The author would like to acknowledge the provision of the Downtown Toronto data set by Optech Inc., First Base Solutions Inc., GeoICT Lab at York University, and ISPRS WG III/4.

\section{Conflicts of Interest}

The author declares no conflicts of interest regarding the publication of this paper.

\section{References}

[1] Tian, B., Wang, L. and Koike, K. (2011) Spatial Statistics of Surface Roughness Change Derived from Multi-Scale Digital Elevation Models. Procedia Environmental Sciences, 7, 252-257. https://doi.org/10.1016/j.proenv.2011.07.044

[2] Hani, M., Fadzil, A., Sathyamoorthy, D. and Asirvadam, V.S. (2011) A Method for Computation of Surface Roughness of Digital Elevation Model Terrains via Multiscale Analysis. Computers \& Geosciences, 37, 177-192. https://doi.org/10.1016/j.cageo.2010.05.021

[3] Brasington, J., Vericat, D. and Rychkov, I. (2012) Modeling River Bed Morphology, Roughness, and Surface Sedimentology Using High Resolution Terrestrial Laser Scanning. Water Resources Research, 48, W11519.

https://doi.org/10.1029/2012wr012223

[4] Grohmann, C.H., Smith, M.J. and Riccomini, C. (2011) Multi-Scale Analysis of Topographic Surface Roughness in the Midland Valley, Scotland. IEEE Transactions on Geoscience and Remote Sensing, 49, 1200-1213. https://doi.org/10.1109/tgrs.2010.2053546

[5] Grohmann, H., Smith, M.J., C.H., and Riccomini, C. (2009) Surface Roughness of Topography: A Multi-Scale Analysis of Landform Elements in Midland Valley, Scotland. Proceedings of Geomorphometry, Zurich, Switzerland, 31 August-2 September 2009. 
[6] Shepard, M.K., Campbell, B.A., Mark, H., Bulmer, M.H., Farr, T.G., Gaddis, L.R. and Plaut, J.J. (2001) The Roughness of Natural Terrain: A Planetary and Remote Sensing Perspective. Journal of Geophysical Research, 106, 32777-32795. https://doi.org/10.1029/2000je001429

[7] Verma, A.K. and Bourke, M.C. (2019) A Method Based on Structure-From-Motion Photogrammetry to Generate Sub-Millimetre-Resolution Digital Elevation Models for Investigating Rock Breakdown Features. Earth Surface Dynamics, 7, 45-66. https://doi.org/10.5194/esurf-7-45-2019

[8] Smith, M.W., Quincey, D.J., Dixon, T., Bingham, R.G., Carrivick, J.L., Irvine-Fynn, T.D.L. and Rippin, D.M. (2016) Aerodynamic Roughness of Glacial Ice Surfaces Derived from High-Resolution Topographic Data. Journal of Geophysical Research: Earth Surface, 121, 748-766. https://doi.org/10.1002/2015jf003759

[9] Hodge, R., Brasington, J. and Richards, K. (2009) Analysing Laser-Scanned Digital Terrain Models of Gravel Bed Surfaces: Linking Morphology to Sediment Transport Processes and Hydraulics. Sedimentology, 56, 2024-2043. https://doi.org/10.1111/j.1365-3091.2009.01068.x

[10] Taud, H. and Parrot, J.-F. (2005) Measurement of DEM Roughness Using the Local Fractal Dimension. Géomorphologie: Relief, Processus, Environnement, 4, 327-338. https://doi.org/10.4000/geomorphologie.622

[11] Zheng, X. and Zhao, K. (2010) A Method for Surface Roughness Parameter Estimation in Passive Microwave Remote Sensing. Chinese Geographical Science, 20, 345-352. https://doi.org/10.1007/s11769-010-0407-3

[12] Hebeler, F. and Purves, R.S. (2007) Modeling DEM data Uncertainties for Monte Carlo Simulations of Ice Sheet Models. 5th International Symposium on Spatial Data Quality, ITC, Enschede, 13-17 June 2007.

[13] Ochoa-Tejeda, V. and Parrot, J.-F. (2017) Slope-Independent Landscape Roughness Attribute Provided by Measurement of Local Contour Line Density. Geoinformatics Geostatistics: An Overview, 5. https://doi.org/10.4172/2327-4581.1000159

[14] Tay, L.T. and Teng, H.-T. (2008) Roughness Index and Fractal Dimension for Surface Information Extraction. Asian Journal of Geoinformatics, 8, 21-24.

[15] Dinesh, S. (2008) Computation of Surface Roughness of Mountains Extracted from Digital Elevation Models. Journal of Applied Sciences, 8, 262-270. https://doi.org/10.3923/jas.2008.262.270

[16] Korzeniowska, K. and Korup, O. (2016) Mapping Gullies Using Terrain-Surface Roughness. AGILE, Helsinki, 14-17 June 2016.

[17] Fisher, P.F. and Tate, N.J. (2006) Causes and Consequences of Error in Digital Elevation Models. Progress in Physical Geography, 30, 467-489. https://doi.org/10.1191/0309133306pp492ra

[18] Gallay, M., Lloyd, C. and McKinley, J. (2010) Using Geographically Weighted Regression for Analyzing Elevation Error of High-Resolution DEMs. Accuracy 2010 Symposium, Leicester, UK.

[19] Thomsen, L.M., Baartman, J.E.M., Barneveld, R.J., Starkloff, T. and Stolte, J. (2015) Soil Surface Roughness: Comparing Old and New Measuring Methods and Application in a Soil Erosion Model. Soil, 1, 399-410.

https://doi.org/10.5194/soil-1-399-2015

[20] Fan, L. and Atkinson, P.M. (2018) A New Multi-Resolution Based Method for Estimating Local Surface Roughness from Point Clouds. ISPRS Journal of Photogrammetry and Remote Sensing, 144, 369-378. 
https://doi.org/10.1016/j.isprsjprs.2018.08.003

[21] Mora, O.E., Lenzano, M.G., Toth, C.K., Grejner-Brzezinska, D.A. and Fayne, J.V. (2018) Landslide Change Detection Based on Multi-Temporal Airborne LiDAR-Derived DEMs. Geosciences, 8, 23.

https://doi.org/10.3390/geosciences 8010023

[22] Höfle, B., Vetter, M., Pfeifer, N., Mandlburger, G. and Stötter, J. (2009) Water surface Mapping from Airborne Laser Scanning Using Signal Intensity and Elevation Data. Earth Surface Processes and Landforms, 34, 1635-1649 https://doi.org/10.1002/esp.1853

[23] Frankel, K.L. and Dolan, J.F. (2007) Characterizing Arid Region Alluvial Fan Surface Roughness with Airborne Laser Swath Mapping Digital Topographic Data. Journal of Geophysical Research, 112, F02025.

https://doi.org/10.1029/2006jf000644

[24] Dorn, H., Vetter, M. and Höfle, B. (2014) GIS-Based Roughness Derivation for Flood Simulations: A Comparison of Orthophotos, LiDAR and Crowdsourced Geodata. Remote Sensing, 6, 1739-1759. https://doi.org/10.3390/rs6021739

[25] Lia, Y., Hub, X., Guanc, H. and Liud, P. (2016) An Efficient Method for Automatic Road Extraction Based on Multiple Features from LiDAR Data. The International Archives of the Photogrammetry, Remote Sensing and Spatial Information Sciences, XLI-B3, 289-293. https://doi.org/10.5194/isprs-archives-xli-b3-289-2016

[26] Rottensteiner, F., Sohn, G., Gerke, M. and Wegner, J.D. (2013) ISPRS Test Project on Urban Classification and 3D Building Reconstruction. ISPRS-Commission III-Photogrammetric Computer Vision and Image Analysis, Working Group III/4-3D Scene Analysis. http://www.commission3.isprs.org/wg4/ 